\title{
Improved cooling algorithm for gauge theories
}

\author{
Philippe de Forcrand ${ }^{\mathrm{a}}$, Margarita García Pérez ${ }^{\mathrm{b} *}$ and Ion-Olimpiu Stamatescu ${ }^{\mathrm{c}}$ \\ ${ }^{a}$ IPS, ETH-Zürich, CH-8092 Zürich, Switzerland \\ bInstituut Lorentz, Rijksuniversiteit Leiden, PO Box 9506, NL-2300 RA Leiden, Nederland \\ ${ }^{\mathrm{c}}$ FEST, Schmeilweg 5, D-69118 Heidelberg, Germany \\ and \\ Inst. Theor. Physik, Univ. Heidelberg, D-69120 Heidelberg, Germany
}

\begin{abstract}
We propose and study a "gold-washing" - type of algorithm which smooths out the short range fluctuations but leaves invariant instantons above a certain size. The algorithm needs no monitoring or calibration.
\end{abstract}

\section{THE COOLING PROBLEM}

As a method to eliminate UV noise and permit studying topological excitations cooling must fulfill certain requirements:

- smooth out the short range fluctuations, including "dislocations"

- preserve the structure at the physical scales, including size and location of instantons

- ensure stability of the cooled structures

- need no monitoring or any engineering which could make it configuration dependent or would only slow it down without ensuring stability.

Since Wilson action has no stable instantons (they shrink and decay under cooling) one usually attempts to calibrate or engineer the cooling procedure based on it such as to obtain metastability, after the noise has been reduced but before the instantons shrank to zero. This, however, makes cooling more an art than a method and also does not answer the question of preserving the physical scales of the original configuration. A general tree level analysis of various actions [1] distinguishes between "under - improved" (e.g., Wilson) and "over - improved" ones; under the former instantons shrink, under the latter instantons beyond a certain size grow [1,2]. We here consider the problem of "improved" cooling algorithms which ensure a high degree of scale invariance for the instantons beyond the short range scale. They

\footnotetext{
${ }^{*}$ MEC and FOM support is thankfully acknowledged.
}

should be useful not only for producing good susceptibility data, but also for studying various conjectured effects of instantons for the spectrum or the chiral transition by providing smooth configurations which preserve the large scale structure of the original (hot) ones. This is why we called this approach "gold washing".

The question of a good cooling algorithm is of course related to that of an action possessing scale invariant classical solutions. "Fix point perfect actions", expected to have generally good scaling properties have been constructed in terms of many loops and higher representations [3]. One still needs, however, to test their usefulness for cooling algorithms in gauge theories.

Our approach here has been different. Since we are primarily interested in instantons our construction refers directly to them. We start with tree level improvement and tune the action to obtain stable instanton solutions which are practically scale invariant beyond some smallsize threshold; the latter should be such that only physically relevant instantons are preserved while short distance topological fluctuations ("dislocations") are eliminated in the cooling. It turns out that the improved cooling algorithm which will be presented below fulfills most of the requirements stated above. The instantons stabilized by it are well fitted by the continuum ansatz for volume integrated quantities (we use twisted b.c. in "time" but the same should hold if the time extension 
of the lattice is large enough). They have integer topological charge within $\mathcal{O}(0.1 \%)$. Higher charge configurations are well behaved under this algorithm, however instanton-antiinstanton (I-A) pairs annihilate of course after a certain number of steps, due to their interaction. The study of pairs necessitates therefore further developments. Although we concentrate here on the cooling problem, the good scaling properties of the actions we study make them useful also as improved Monte Carlo actions. While our analysis here refers explicitely to $S U(2)$, most relations are valid for $S U(N)$ generally.

\section{IMPROVED COOLING}

One can take various loops in the action and tune their couplings to improve the approach to continuum. For simplicity we work only with fundamental, planar loops of size $m \times n$ :

$$
\begin{aligned}
S_{m, n} & =\sum_{x, \mu, \nu} \operatorname{Tr}\left(1-\frac{1}{2}\left(\nu_{x}^{\square}+\nu \underset{x^{\square}}{\square}\right)\right)(1) \\
S & =\sum_{i=1}^{5} c_{i} \frac{1}{m_{i}^{2} n_{i}^{2}} S_{m_{i}, n_{i}}
\end{aligned}
$$

Here $\left(m_{i}, n_{i}\right)=(1,1),(2,2),(1,2),(1,3),(3,3)$ for $i=1, \ldots, 5$. The Gibbs factor is $\exp \left(-\frac{1}{g^{2}} S\right)$. The choice

$S(\epsilon): \quad c_{1}=(4-\epsilon) / 3, c_{2}=(\epsilon-1) / 3$

$c_{3,4,5}=0$, leads to "under-improved" actions for $\epsilon>0$ (instantons shrink, as for the Wilson action $\epsilon=1)$. For $\epsilon<0$ the actions are "overimproved" (instantons grow) and for $\epsilon=0$ there are no $\mathcal{O}\left(a^{2}\right)$ corrections [1]. Using the results in [1] one can construct a one parameter set of actions that have no $\mathcal{O}\left(a^{2}\right)$ and $\mathcal{O}\left(a^{4}\right)$ corrections

$c_{1}=\left(19-55 c_{5}\right) / 9, \quad c_{2}=\left(1-64 c_{5}\right) / 9$

$c_{3}=\left(-64+640 c_{5}\right) / 45, \quad c_{4}=1 / 5-2 c_{5}$

The simplest ones are $S(4 L i)\left(c_{5}=0\right)$ and $S(3 L i)\left(c_{5}=1 / 10\right)$. Since for the configurations we have studied numerically the former tends to under-improve while the latter over-improves, we chose for our simulations a combination of both, $S(5 L i)$, with $c_{5}=1 / 20$. This action showed already good properties and we did not try to optimize it further. The cooling algorithm exactly minimizes the above local action at each step and involves no further calibration or engineering.

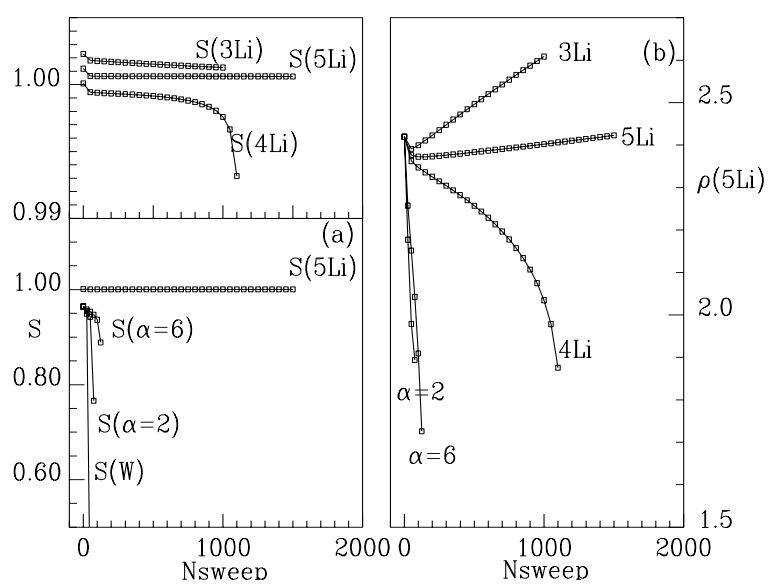

Figure 1. Action (a) and size (b) vs sweep number for various algorithms. $\mathrm{S}(\mathrm{W})$ is Wilson action.

We first check the behaviour of various instantons under the improved cooling taking as starting configurations those obtained by cooling with $S(\epsilon)$ with various $\epsilon$. All results are obtained on $12^{4}$ lattices and for gauge group $S U(2)$. Since we wanted to disentangle small distance from finite lattice size effects, we use throughout twisted p.b.c. with $k=(1,1,1)$ twist, to ensure that instanton solutions exist on finite lattices [4.11. Fig. 1 compares the cooling behaviour of some algorithms $(S(\alpha)$ denote the underrelaxed algorithms of [5]): (a) the action (notice the different scales) and (b) the size $\rho_{\text {peak }}=6 \pi^{2} N_{s} /\left.S(t)\right|_{\max }$ for the same instanton, as function of the cooling step $\left(N_{s}=12\right.$ here). Fig. 2 shows the behaviour of instantons of various initial sizes under the $5 \mathrm{Li}$ cooling. As it can be seen, they remain practically unchanged over any practicable number of cooling sweeps and for $\rho>\rho_{0} \simeq 2.3$ (in units of a), which seems to represent a stability threshold for this cooling algorithm. The size of the original instanton has been varied by applying $S(\epsilon)$ cooling, therefore these instantons do not correspond to minima of $S(5 L i)$. Submitting such configurations to $5 L i$ cooling first readapts them to the new equations of motion, implying the small changes which can be observed in the first $\simeq 50$ sweeps 


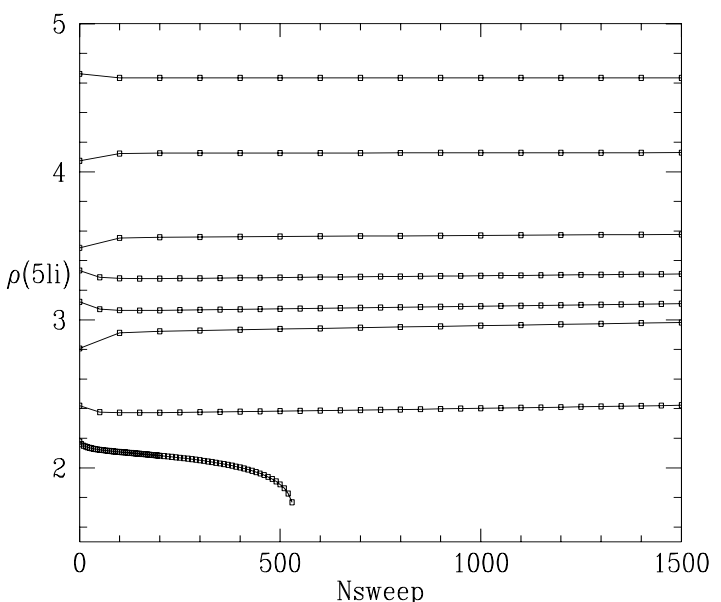

Figure 2. Instanton size under $5 L i$ improved cooling starting from various configurations.

of Fig. 2. Fig. 3 shows the effect of $5 L i$ cooling on instantons of different sizes taken from various stages of Wilson cooling of 3 different starting configurations (the upper curves give the values of $S(5 L i)$ for these Wilson cooled instantons). We see how the $S(5 L i)_{\min }$ emerges as an envelope. We observe again the stability threshold around 2.3 and the extent of configuration dependence at small $\rho$. The triangles are data from Monte Carlo instantons $\left(\beta=2.5,12^{4}\right.$ lattice) cooled with 300 sweeps of $5 \mathrm{Li}$ cooling.

To describe the instantons we use the volume integrated action and charge densities ("profiles"):

$$
\begin{aligned}
S(t) & =N_{s} \sum_{x, y, z} S(x, y, z, t) \\
Q(t) & =8 \pi^{2} N_{s} \sum_{x, y, z} Q(x, y, z, t)
\end{aligned}
$$

to be fitted by the continuum ansatz with periodicity satellites, e.g. for the charge:

$$
\begin{gathered}
Q(t)= \pm 6 \pi^{2} N_{s} \rho^{4}\left[f(t)+f\left(t+N_{t}\right)+f\left(t-N_{t}\right)\right] \\
f(t)=\left[\left(t-t_{0}\right)^{2}+\rho^{2}\right]^{-5 / 2}
\end{gathered}
$$

Here $t_{0}$ defines the location and $\rho$ the width, " $\rho_{\text {profile }}$ ". The latter agrees rather well with $\rho_{\text {peak }}$ as long as the total charge is \pm 1 . This is ensured at not too small $\rho$, i.e. for stable instantons.

Since Wilson action produces short range fluctuations one can ask whether the effective cut-off at $\rho_{0} \simeq 2.3$ ensures the absence of unphysical fluc-

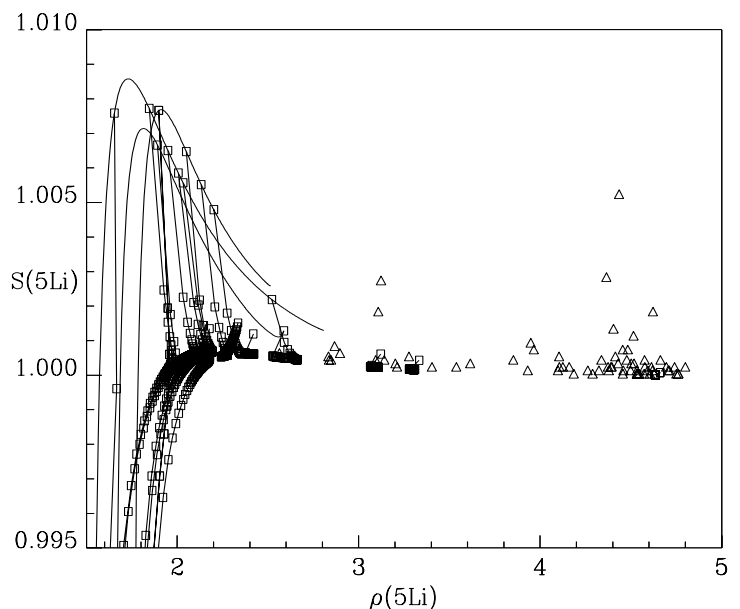

Figure 3. Instanton evolution (action and size) during $5 L i$ cooling.

tuations in cooled MC configurations. Following an argument of Pugh and Teper [6] we write the contribution of small instantons as $(N=2$ here)

$$
\begin{array}{r}
{\left[\rho_{0}(a) a\right]^{-4} \mathrm{e}^{-\beta S_{W}\left(\rho_{0}\right)}} \\
a(\beta) \simeq \Lambda^{-1} \mathrm{e}^{-\frac{\beta}{4 N \beta_{1}}} ; \quad \beta_{1}=\frac{11 N}{48 \pi^{2}}
\end{array}
$$

We can calculate $S_{W}\left(\rho_{0}\right)$ by cooling large instantons with the Wilson action (they shrink). One can then find $\rho_{0}(a(\beta))$ from the condition that the two factors in Eq.(8) compensate each other such that the contribution from instantons larger than $\rho_{0}$ stays finite in continuum. We obtain a very flat dependence: $\rho_{0}=1.9276,1.9280,1.9284$ for $\beta=3,6, \infty$, respectively. Hence a threshold $\rho_{0}$ of about 2 and independent on $\beta$ seems satisfactory.

\section{MONTE CARLO ANALYSIS}

To illustrate the capability of improved cooling we apply $5 L i$ cooling to a Monte Carlo simulation at $\beta=2.5$ on a $12^{4}$ lattice, $k=(1,1,1)$ twist. We use 160 configurations generated by heat bath with Wilson action and taken 250 sweeps apart (after 20000 thermalization sweeps). In Fig. 4 we show results from 300 cooling sweeps. The stable situation typically sets in between 50 and 100 sweeps. Instantons very near below the threshold $\rho_{0} \simeq 2.3$ may need more sweeps to disappear - see Fig. 2; this introduces a systematic error 

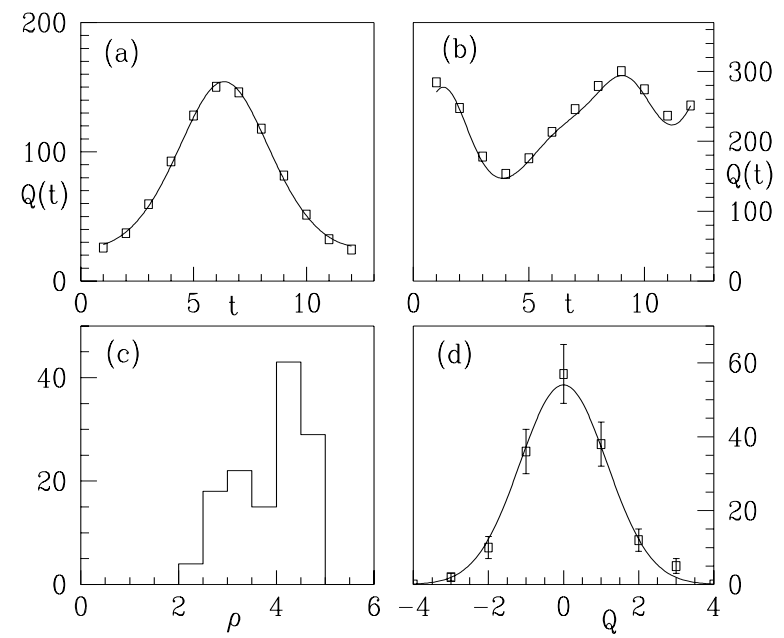

Figure 4. MC analysis: typical charge 1 and 3 configurations and size and charge distribution.

in the number of stable instantons in this region, which vanishes with increasing number of sweeps. In Fig. 4a,b we show two typical configurations, the first with one wide ( $\left.\rho_{\text {profile }}=4.66\right)$ instanton and the second with total charge 3 showing 3 instantons of widths $2.80,4.47$ and 3.37 located at $\mathrm{t}=1.22,6.22$ and 9.29 , together with the fit Eq.(7). In Fig. 4c we show the size and in $4 \mathrm{~d}$ the charge distributions. The latter is representative for physical instantons, i.e. beyond the threshold $\rho_{0}$. A gaussian fit $\exp \left(-b Q^{2}\right)$ gives $b=0.34$ at cooling sweep 20 and $b=0.37$ beyond 100 . The charge stabilizes to within $1 \%$ an integer between 20 and 100 sweeps, therefore susceptibility and charge distribution can be estimated quite early. The topological susceptibility extracted from data at 300 sweeps is $Q^{2} / 12^{4}=6.8(9) 10^{-5}$. The size distribution, however, represents only the stable situation and does not take into account I-A pairs which would have annihilated before. For illustration we show in Fig. 5 the charge and action history (a) and the evolution of a typical configuration under the $5 L i$ cooling $(\mathrm{b}, \mathrm{c})$. The initial structure seems to have a double instanton and 2 I-A pairs. An I-A pair can still be seen with the continuum ansatz Eq. (7) at sweep 5 (sizes 1.38 and 2.95) and at sweep 10 (sizes 2.74 and 3.36), together with the double instanton (size 2.5 at sweep 5 and 2.86 at 10) - see Fig. 5b. After

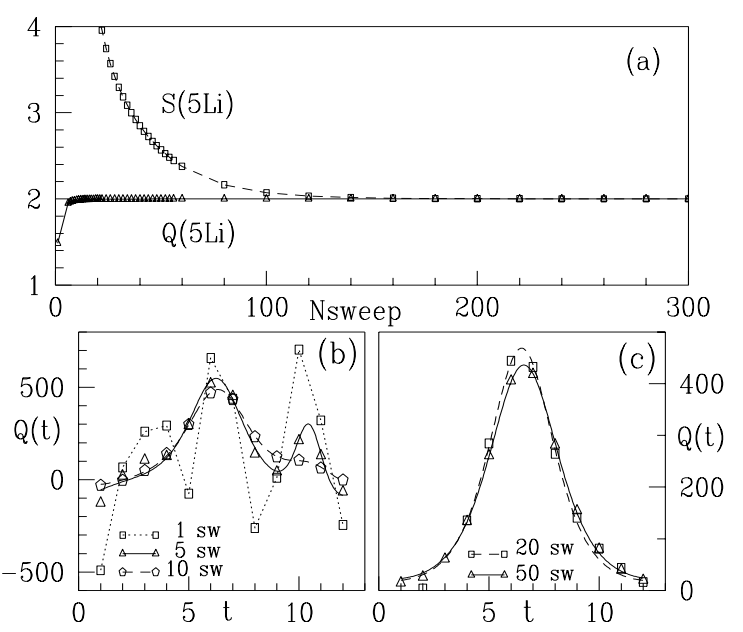

Figure 5. Early evolution of a MC conf.. The line for $1 \mathrm{sw}$ is an eye-guide, the rest fits based on (7).

sweep 20 (Fig. 5c) only the latter remains (size: 3.07 at $20,3.29$ at 50, 3.52 at 300 sweeps). Hence this annihilation may result in a depletion of the lower part of the size distribution. The question of describing complex configurations is a separate problem currently under study.

We are very indebted to Pierre van Baal and Jeroen Snippe for discussions and suggestions. MGP and IOS profitted very much from the participation to the Benasque Center of Physics which gave the opportunity to work concentrated on this subject. Use of the Fujitsu VP600 of the University of Karlsruhe and a NCF grant for use of the Cray C98 are thankfully acknowledged.

\section{REFERENCES}

1. M. García Pérez, A. Gonzalez-Arroyo, J. Snippe and P. van Baal, Nucl. Phys. B413 (1994) 535.

2. Ph. de Forcrand and S. Kim, in this volume.

3. T. DeGrand, A. Hasenfratz, P. Hasenfratz and F. Niedermayer, Prep. BUTP-95/14,15.

4. P.J. Braam and P. van Baal, Commun. Math. Phys. 122 (1989) 267.

5. C. Michael and P.S. Spencer, HU TFT 95-21; Phys. Rev. D50 (1994) 7570.

6. D.J.R. Pugh and M. Teper, Phys. Lett. B 224 (1989) 159. 